\title{
Pelli-Robson Contrast Sensitivity Eye Chart
}

National Cancer Institute

\section{Source}

National Cancer Institute. Pelli-Robson Contrast Sensitivity Eye Chart. NCI Thesaurus.

Code $C 117878$.

A diagrammatic method to test visual acuity and contrast sensitivity, which utilizes large letters (at a 20/60 acuity) that decrease in contrast from right to left and top to bottom. 